\title{
Detection and Correction of Abnormal Pixels in Hyperion Images
}

\author{
T. $\operatorname{Han}^{1}$, D. G. Goodenough ${ }^{1,2}$, A. Dyk ${ }^{2}$, and J. Love ${ }^{1,2}$ \\ ${ }^{1}$ University of Victoria, Victoria, Canada \\ ${ }^{2}$ Pacific Forestry Centre, Natural Resources Canada, Victoria, Canada \\ 3800 Finnerty Road, Victoria, BC, Canada, V8P 5C2 \\ (250 - 363 -6018, office); than@uvic.ca
}

\begin{abstract}
Hyperion images are currently processed to level 1 a (from level 0 or raw data). These level 1a images are files of radiometrically corrected data in units of either watts/(sr $\times$ micron $\left.\times \mathbf{m}^{2}\right) \times \mathbf{4 0}$ for VNIR bands or watts/(sr $\times$ micron $\left.\times \mathbf{m}^{2}\right)$ $\times 80$ for SWIR bands. Each distributed Hyperion level 1a image tape contains a log file, called "(EO-1 identifier).fix.log", that reports the bad or corrupted pixels (called known bad pixels) found during the pre-flight checking, and details how they were fixed. All bad pixels should be corrected in a level 1a image. However, bad pixels are still evident. In addition, there are dark vertical stripes in the image that are not reported in the $\log$ file. In this paper, we introduce a method to detect and correct the bad pixels and vertical stripes (we will refer to these occurrences as abnormal pixels). Images from the Greater Victoria Watershed and other EVEOSD test sites are used to determine how stationary the locations of the abnormal pixels are. After abnormal pixel correction a Hyperion image is ready for geometric correction, atmospheric correction, and further analysis.
\end{abstract}

\section{INTRODUCTION}

EO-1's Hyperion sensor provides a new class of earth observation data for improved Earth surface characterization [1]. As part of NASA's EO-1 program, the research reported on in this paper is being conducted under the Evaluation and Validation of EO-1 for Sustainable Development (EVEOSD) project. The EVEOSD project is investigating the determination of canopy chemistry from hyperspectral data and the improvements in forest classification possible with hyperspectral remote sensing. It is therefore important that any artifacts in the Hyperion imagery be removed.

Hyperion acquires data in pushbroom mode with two spectrometers, one in the visible and near infrared (VNIR) range and another in the short-wave infrared (SWIR) range. The highest level of the distributed Hyperion dataset is called Hyperion level 1a, which is radiometrically-corrected. The main specifications for the Hyperion level la data set are listed in Table 1.

The Hyperion level 1a dataset is generated from its level 0 dataset through several processing steps including: smear correction, echo correction, background removal, radiometric correction, bad pixel repair, and image quality checking [1]. For each processing step, there is a corresponding log file or metadata file created to indicate what intermediate processing has occurred. The bad pixel repair metadata file
(MD10) reports the bad pixel locations, both spatially and spectrally, in the level 0 dataset, and specifies how they were fixed. Once the level 1a dataset is generated, there should be no bad or corrupted pixels remaining. However, after a visual examination of a Hyperion level la dataset, it was apparent that bad pixels still remained. Furthermore, it was discovered that the positions of some of the bad pixels are 11 pixels to the left of the bad pixel positions recorded in the MD10 file. Finally, there were dark vertical stripes in the image that are not recorded in the log file. The abnormal pixels must be identified and corrected before further analysis of an image can proceed.

\section{TABLE 1}

SPECIFICATIONS OF HYPERION LEVEL 1A DATASET

\begin{tabular}{|l|l|}
\hline Wavelength & $356-2577(\mathrm{~nm})$ \\
\hline Number of Bands & 242 \\
\hline Pixel Size & $30(\mathrm{~m})$ \\
\hline Image Dimension & $256 \times 6460$ \\
\hline VNIR Bands & $1-70(356-1058 \mathrm{~nm})$ \\
\hline SWIR Bands & $71-242(852-2577 \mathrm{~nm})$ \\
\hline Data Type & 2 Signed Integers \\
\hline Pixel Order & BIL \\
\hline Byte Order & Network (IEEE) \\
\hline File Size & $800,427,520$ (bytes) \\
\hline
\end{tabular}

\section{ABNORMAL PIXEL CLASSIFICATION}

The majority of abnormal pixels in Hyperion level 1a images appear as dark stripes, either continuous or intermittent through columns. The image we use here is a subset image of our main test site - Greater Victoria Watershed (GVWD), which was acquired on Sept. 10, 2001. According to their appearance and DN values, the abnormal pixels can be separated into four distinct classes:

- Class 1 - continuous with atypical DN values.

These abnormal pixels have atypical DN values-usually manifested as extremely small DN values, such as -32768 . An example is given in Fig. 1. 
- Class 2 - continuous with constant DN values.

These abnormal pixels have constant DN values in an entire column. An example is displayed in Fig. 2 .

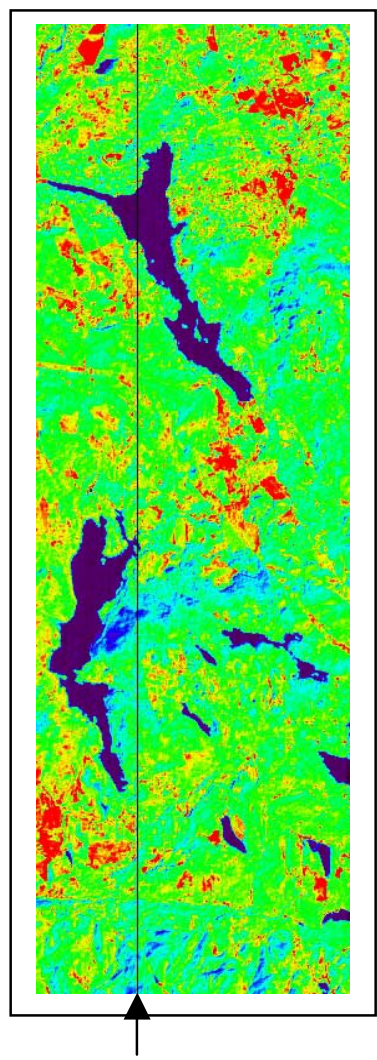

Fig. 1. Continuous abnormal pixels with atypical DN values, band 94

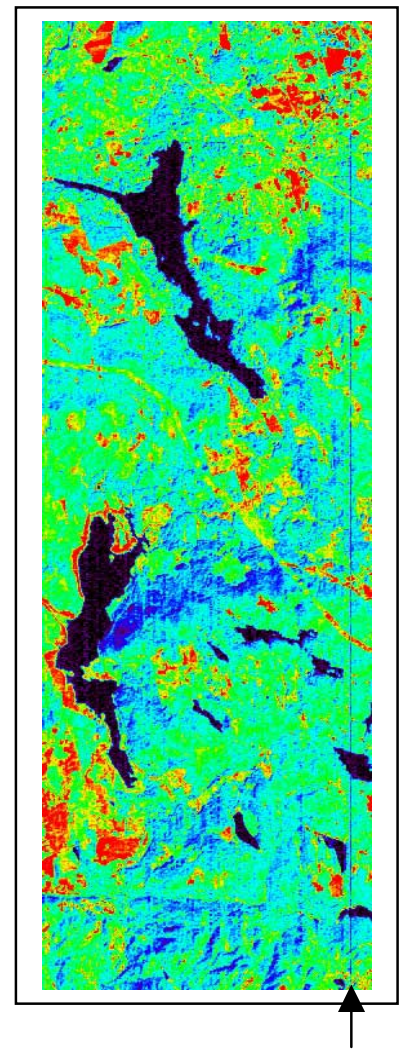

Fig. 2. Continuous abnormal pixels with constant DN values, band 119 .
- Class 3 - Intermittent with atypical DN values.

These pixels do not occur through an entire column. They appear as dark dots with very small DN values, as shown in Fig. 3.

- Class 4 - Intermittent with lower DN values.

This is the most common case. Compared to neighboring pixels, these abnormal pixels have lower DN values. They appear intermittently throughout a column and are not constant in value. An example is given to show them in Fig. 4.

\section{ABNORMAL PIXELS AND SCENE LOCATION}

In order to investigate the abnormal pixels, we created spatial subsets of images from two EVEOSD test sites: Hoquiam in Washington state and the Greater Victoria Watershed District (GVWD) in British Columbia. For each image subset, lines $3700-4500$ were selected. By comparing the equivalent bands of the two datasets, we found that most abnormal pixels are stationary. There are two exceptions.
For class 3 abnormal pixels (intermittent with atypical DN values), the abnormal pixels in the two datasets occurred in the same columns, but at different lines. In images with class 4 pixels, the visibility of abnormal pixels (intermittent with lower DN values) is dependent on neighboring pixels, and consequently it was difficult to find a correlation between the two datasets. Fig. 5 and Fig. 6 are examples of the spatial variability of class 4 pixels.

\section{POSSIBLE CAUSES OF THE ABNORMAL PIXELS}

There are many possible causes for the abnormal pixels, including: detector failure, errors during data transfer, and improper data correction. To narrow down the causes, we compared a Hyperion level 0 dataset of the GVWD test site with a level 1 dataset of the same acquisition. We found that the abnormal pixels in the Hyperion level la dataset were not created by the correction process (from level 0 to level 1a), because the abnormal pixels found in the level 1a dataset also existed in the Hyperion level 0 dataset.

One possible reason for the abnormal pixels could be that the calibration of the detectors in the detector array becomes unbalanced. The Hyperion system acquires data in pushbroom mode, in which there is a separate detector to gather data for each column in the image it generates. If the detectors are not calibrated properly striping artifacts could easily be generated .

Class 1 abnormal pixels (continuous with atypical DN values) could be caused by the SWIR smear and echo correction. During this correction process a small DN value (1) might be increased greatly (-32768) if the correction algorithm does not account for negative values. This could be corrected by setting all negative pixel values to zero after the smear correction, and then proceeding with the echo correction [1].

\section{ABNORMAL PIXEL CORRECTION}

It is straightforward and relatively safe to correct abnormal pixels by replacing them with their nearest neighbors. Class 1 and 3 abnormal pixels can be easily identified by the extremity of their DN values. Class 2 abnormal pixels are also easily discovered, because they appear across an entire column. Class 4 abnormal pixels (intermittent with lower DN values) are more difficult to detect. They are not visually apparent, appear in different lengths, and can be confused with normal pixels and real vertical ground features. However, class 4 abnormal pixels have smaller DN values than that of their immediate left and right neighbors. Furthermore, they tend to run vertically for varying lengths. 


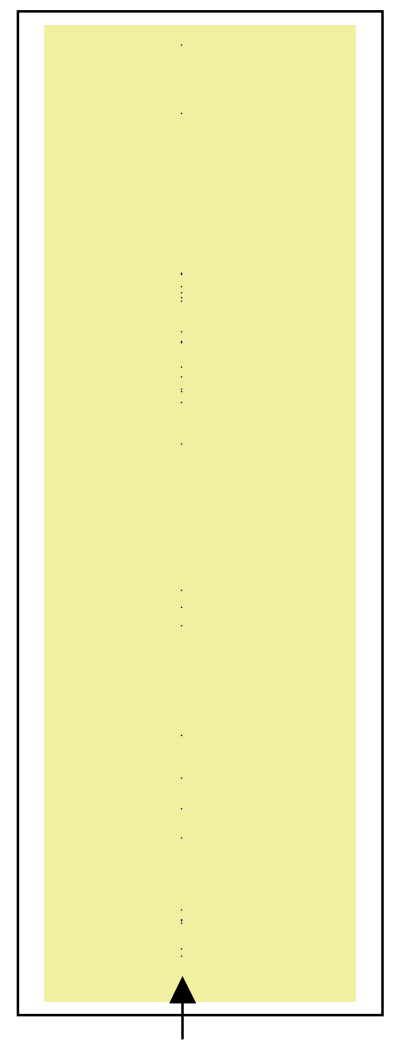

Fig. 3. Intermittent abnormal pixels with atypical $\mathrm{DN}$ values, band 190.

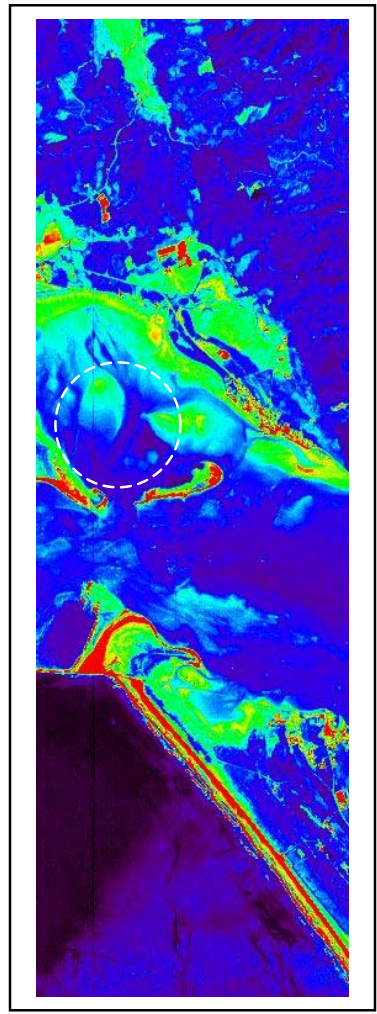

Fig. 5. Band 27 of Hoquiam dataset showing variable class 4 pixels.

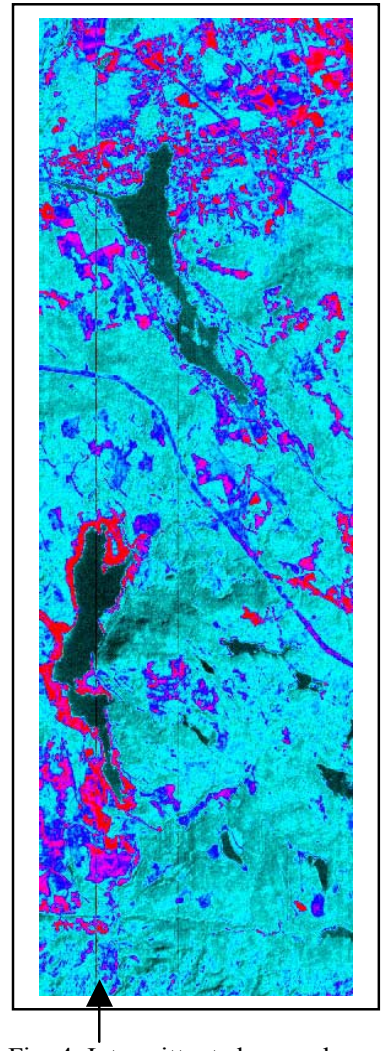

Taking advantage of these two common features, an IDL program was created to automate the abnormal pixel correction process.

The program reads in an image cube by spatial tiling and saves it as a 3D array. It then traverses each band horizontally to compare each pixel's DN value with that of its immediate left and right neighboring pixels. If the pixel's DN value is smaller than the DN values of both neighbors, this pixel is labeled as abnormal. After abnormal labeling, the program traverses each band vertically to count the number of consecutive abnormal pixels and the total number of pixels in each column. If the number of the consecutive abnormal pixels is greater than a user-defined-threshold-value (the longest vertical ground feature in pixels) and if the percentage of abnormal pixels in the column is greater than another user-defined-threshold-value (usually 50\%), then the pixels in the column are marked as abnormal pixels, and their locations are recorded in a mask image file. The final step is to correct the abnormal pixels by replacing their DN values with the average $\mathrm{DN}$ values of their immediate left and right neighboring pixels.

The abnormal pixel detection algorithm works for most bands, except for intensively striped and noisy bands. Examples of the processing results are shown in the following fig. 7, 8, and 9. Table 2 lists the detected abnormal pixels for all classes in the subset of Hoquiam image. These may assist other investigators in analyzing Hyperion data.

\section{Conclusions}

The abnormal pixels in Hyperion level 1a images appear as dark vertical stripes that can be divided into 4 distinct classes: continuous with atypical DN values, continuous with constant DN values, intermittent with atypical DN values, and intermittent with lower DN values. Each class has two common features: they have smaller DN values compared to those of their immediate left and right neighbors, and they all run vertically for a certain length. For classes 1, 2 , and 3 , the abnormal pixels are stationary; i.e. they occur at the same column image to image. For class 4 pixels, the positions of the abnormal pixels can vary from image to image. Abnormal pixels can be effectively detected and corrected with our abnormal pixel correction algorithm. However, in order to correctly identify the abnormal pixels, the threshold value representing the longest vertical feature on the ground needs to be well determined. If the value is exaggerated, some abnormal pixels will be missed. If this value is underestimated, some normal pixels will be treated as abnormal pixels, and information will be lost. 


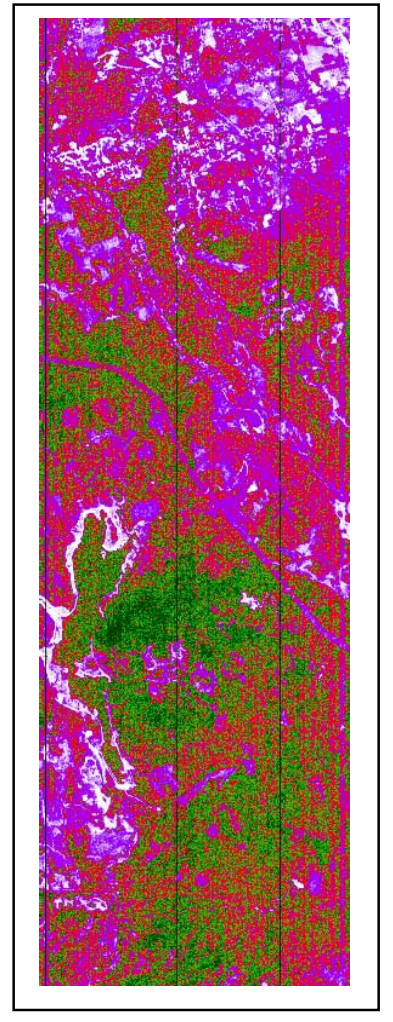

Fig. 7. Before correction, band 11 , GVWD

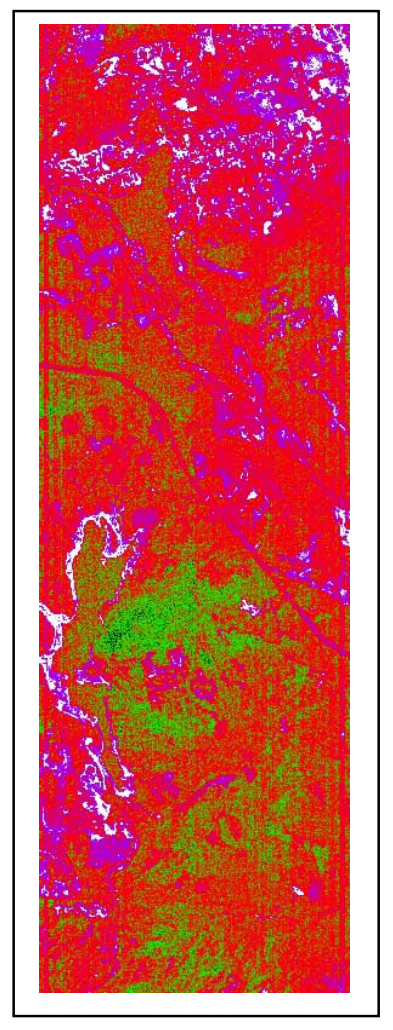
pixels detected.
TABLE 2

LOCATIONS OF THE DETECTED ABNORMAL PIXELS

\begin{tabular}{|c|c|c|c|}
\hline Band & Column & Band & Column \\
\hline 5 & 114,141 & 57 & 13 \\
\hline 6 & $6,68,172$ & 75 & 2 \\
\hline 7 & $7,68,179,185$ & 94 & 82 \\
\hline 8 & $7,12,68,114,121$ & 99 & 81 \\
\hline 9 & $6,68,114$ & 116 & 127 \\
\hline 10 & $6,114,131,199$ & $119-120$ & 240 \\
\hline 11 & $6,114,199$ & 121 & 196 \\
\hline 12 & 6,114 & 125 & $54,115,160,164$ \\
\hline 13 & 114 & 127 & $40,66,213$ \\
\hline 14 & 114,247 & 128 & $30,96,126$ \\
\hline $15-26$ & 114 & 165 & 148 \\
\hline $27-28$ & 47,114 & 168 & 245 \\
\hline $29-34$ & 114 & 169 & 12,23 \\
\hline 39 & 177 & 190 & 113 \\
\hline 40 & 13 & $200-201$ & 8 \\
\hline $48-51$ & 20 & 203 & 104,115 \\
\hline 52 & 13,33 & 222 & 98 \\
\hline 53 & 33 & 225 & 186 \\
\hline
\end{tabular}

ACKNOWLEDGMENTS

The authors would like to acknowledge the support of NASA's EO-1 Project, the Canadian Space Agency, the Pacific Forestry Centre of Natural Resources Canada, and the Department of Computer Science at the University of Victoria. We are also grateful for the support of the Natural Sciences and Engineering Research Council. Ground reference data collections were supported by the Greater Victoria Watershed District and Weyerhaeuser Corporation.

\section{REFERENCES}

[1] TRW, "EO - 1 Hyperion Document," 2001.

[2] J. A. Richards and X. Jia, Remote Sensing Digital Image Analysis - An Introduction, 3rd ed. Germany: Springer, 1999.

[3] J. Nieke, "Imaging Spaceborne and Airborne Sensor Systems in the Beginning of the Next Century," Proc. European Symposium on Aerospace Remote Sensing, 1997, pp. 22-26.

[4] D. W. Fanning, IDL programming Techniques, 2nd ed: Fanning Software Consulting, 2000

Fig. 9. After correction. 